\title{
Heuristic Approaches to Energy-Efficient Network Design Problem
}

\author{
Cigdem Sengul Robin Kravets \\ Department of Computer Science, University of Illinois at Urbana-Champaign \\ sengul@uiuc.edurrhk@cs.uiuc.edu
}

\begin{abstract}
Energy management remains a critical problem in wireless networks since battery technology cannot keep up with rising communication expectations. Current approaches to energy conservation reduce the energy consumption of the wireless interface either for a given communication task or during idling. However, a complete solution requires minimizing the energy spent for both communication (i.e., for data and control overhead) and idling. This problem can be expressed as an energy-efficient network design problem, which is, not surprisingly, NP-hard. Therefore, in this paper, we study three heuristic approaches. Our study shows that the first approach that prioritizes communication energy conservation does not save energy. The second approach, which tries to reduce energy used for both data and in idling, becomes cost-prohibitive due to its high control overhead. Hence, we propose a third approach that prioritizes idling energy conservation. Due to its low control overhead, this approach meets the challenge of operating the network with low energy cost.
\end{abstract}

\section{Introduction}

Energy management is one of the greatest challenges in wireless networks due to the continuous increase in the energy requirements of wireless devices and the slow advancement of battery technology. Therefore, it is essential to incorporate energy efficiency into the design of network protocols. Current research has focused on either communication energy conservation, which aims to optimize the use of the wireless interface for a given communication task, or idling energy conservation, which aims to optimize energy use when the interface is idle. Obviously, a complete solution needs to reduce both communication and idling energy. However, the complex interactions between communication and idling energy conservation techniques present a significant challenge. To this end, this paper explores the limits of traditional stand-alone techniques and exposes some commonly held myths about energy conservation.
Contrary to common expectations [7], joint utilization of communication and idling energy conservation techniques is likely to exhibit negative interactions. Consider two techniques: energy-aware routing using transmission power control (TPC) and power management. TPC allows tuning the transmission power level based on the distance between the sender and the receiver. Due to non-linear power attenuation, transmitting via multiple short hops may consume less energy than via one long hop. Hence, energy-aware routing [14, 23] exploits TPC and routes through short hops to reduce communication energy. Power management, on the other hand, saves idling energy by allowing nodes to switch to a power-save mode (PSM), in which a node spends most of its time in a low-power sleep state $[9,25]$. The negative interaction occurs since energy-aware routing saves energy at the expense of using more relays and power management saves energy by eliminating redundant relays. In this paper, we show how such interactions impact the design of a complete solution to energy conservation.

Minimizing energy consumption due to both communication (i.e., data and control overhead) and idling while satisfying traffic constraints is an energy-efficient network design problem, which is, not surprisingly, NP-hard [24, 22]. The main contribution of our research is an in-depth analysis of three heuristic approaches to this problem: (1) minimizing communication energy use as a primary goal, (2) jointly optimizing for both communication and idling energy use and (3) minimizing idling energy use as a primary goal. Our analysis shows that the first approach is not practical for various wireless cards and the control overhead of the second approach does not support scalable solutions. Our extensive evaluation verifies the superiority of our approach of minimizing idling energy as a primary goal and shows that our approach meets the challenge of operating the network with low energy cost without degrading communication performance.

The rest of the paper is organized as follows. In the next section, we present an energy model to evaluate the energyefficiency of communication and idling energy conservation. In Section 3, we formally define the energy-efficient network design problem and in Section 4, we describe the 
three heuristic approaches in detail. Performance evaluation results are presented in Section 5. Section 6 presents concluding remarks.

\section{Energy-Awareness in Wireless Networks}

To support energy-awareness in wireless networks, it is essential to understand the trade-offs in communication. To this end, we present an energy model based on the characteristics of wireless cards and node participation in the network. Next, we use this model to highlight the limitations of two prominent techniques for communication and idling energy conservation.

\subsection{Energy Model}

The energy consumption of a network is determined by the energy spent by all nodes. The energy consumption of each node $i$ is the sum of its communication energy, $E_{\text {comm }}(i)$, and its idling energy, $E_{\text {passive }}(i)$. Obviously, $E_{\text {comm }}(i)$ and $E_{\text {passive }}(i)$ are strongly tied to the energy characteristics of a wireless card and the node's participation in the network.

The energy characteristics of a wireless card are determined by its operating modes: transmit, receive, idle and sleep. Transmit is the most power-hungry mode and, to compensate for this, current wireless cards support a range of transmit power levels. Sleep power is typically negligible and idle power is as large as receive power [11]. In addition to power, $E_{\text {comm }}(i)$ and $E_{\text {passive }}(i)$ are also a function of the time spent in each state, which in turn depends on many factors, including traffic load, routing decisions and packet failures.

For each node $i, E_{\text {comm }}(i)$ is the sum of the energy consumed for data and control overhead, $E_{\text {data }}(i)$ and $E_{\text {control }}(i)$, respectively. Given the total time spent in data reception, $t_{r x}^{\text {data }}(i)$, and the time spent transmitting to each next hop node $j(j \in N H), t_{t x}^{d a t a}(i, j)$ :

$$
E_{\text {data }}(i)=\sum_{j \in N H} t_{t x}^{\text {data }}(i, j) \cdot P_{t x}(i, j)+t_{r x}^{\text {data }}(i) \cdot P_{r x},
$$

where the receive power is $P_{r x}$. The transmission power, $P_{t x}(i, j)=P_{\text {base }}+P_{t}(i, j)$, where $P_{b a s e}$ is the base transmitter cost and $P_{t}(i, j)$ is the transmit power level. $P_{t}(i, j)$ attenuates with the $n^{\text {th }}$ power of the distance between nodes $i$ and $j$, where $2 \leq n \leq 4$, depending on channel characteristics. Since $P_{r x}$ and $P_{b a s e}$ are fixed costs, $E_{\text {data }}(i)$ is determined by how much data a node relays and the $P_{t x}(i, j)$ 's.

Given the time spent in transmitting and receiving control packets, $t_{r x}^{c t r l}$ and $t_{t x}^{c t r l}$, respectively:

$$
E_{\text {control }}(i)=t_{t x}^{c t r l}(i) \cdot P_{t x}^{\max }+t_{r x}^{c t r l}(i) \cdot P_{r x} .
$$

We assume that control packets are transmitted with maximum power level, $P_{t}^{\max }$, and so use $P_{t x}^{\max }$ for transmission. From (2), it is easy to see that $E_{\text {control }}$ can only be reduced by limiting control overhead.

$E_{\text {passive }}(i)$ represents the energy consumed when a node is not involved in reception or transmission. During this time, a node can be in a sleep state with sleep power, $P_{\text {sleep }}$, for a duration of $t_{\text {sleep }}$ or in an idle state with idle power, $P_{\text {idle }}$, for a duration of $t_{\text {idle }}$. Therefore,

$$
E_{\text {passive }}(i)=t_{\text {idle }}(i) \cdot P_{\text {idle }}+t_{\text {sleep }}(i) \cdot P_{\text {sleep }}+E_{\text {sw }} \text {, }
$$

where $E_{s w}$ captures the energy cost of switching states. Obviously, $E_{\text {passive }}(i)$ is minimized if the node stays mostly in sleep state.

Based on this node-based energy model, the network energy consumption, $E_{\text {network }}$, is defined as:

$$
E_{\text {network }}=\sum_{i=1}^{m} E_{\text {comm }}(i)+E_{\text {passive }}(i),
$$

where $m$ is the number of nodes. Using this energy model, we next elaborate communication and idling energy conservation.

\subsection{Communication vs. Idling Energy Conservation}

Energy conservation in wireless networks targets the energy spent in specific modes of a wireless card. Due to the high power of transmit mode, much research has been directed at minimizing $P_{t}(i, j)$ via transmission power control. For example, energy-aware routing (e.g., PARO [14], MTPR (Minimum Transmission Power Routing) [23]) uses $P_{t}(i, j)$ as a routing metric. However, the main goal is to save energy in terms of $\sum_{i} E_{\text {data }}(i)$, and the effects on $\sum_{i} E_{\text {control }}(i)$ and $\sum_{i} E_{\text {passive }}(i)$ are ignored.

However, $E_{\text {passive }}(i)$ is the dominating energy consumer when there is no communication [11]. To reduce $E_{\text {passive }}(i)$, power management is typically used. Power management provides two modes, power-save mode (PSM) and active mode (AM). In PSM, a node keeps its wireless card mostly in sleep state (e.g., IEEE PSM [5]). In AM, a node is either transmitting, receiving or idling. While PSM may benefit lightly-loaded networks, it severely limits network capacity as the load increases [25]. Therefore, power management allows transitions between PSM and $\mathrm{AM}$, which can be triggered based on traffic duration (e.g., ODPM (On-Demand Power Management) [25]) or topology (e.g., Span [9]) or both (e.g., TITAN [21]). However, power management only optimizes for $\sum_{i} E_{\text {passive }}(i)$ and ignores $\sum_{i} E_{\text {comm }}(i)$.

Both energy-aware routing and power management optimize for different radio states in isolation. Next, we study the joint utilization of these approaches as an energyefficient network design problem. 


\section{Energy-Efficient Network Design Problem}

The energy-efficient network design problem is similar to traditional network design problems, which, given a weighted graph, ask for a subgraph of minimum total weight satisfying some connectivity requirements [13]. In this section, we give a formal definition of the problem, which is NP-hard.

We model a wireless network as an undirected graph. Associated with each source-destination pair $\left(s_{i}, d_{i}\right)$, there is a non-negative traffic demand $r_{i}$. Each node and edge are assigned weights, $c(v)$ and $w(e)$, respectively. While $c(v)$ is either $P_{\text {idle }}$ or $P_{\text {sleep }}$ based on the power management state of a node $v, w(e)$ is determined by $P_{t x}(e)$ and $P_{r x}$. Based on this notation, the energy-efficient network design problem is defined as follows:

Definition 1 Given a network, the goal of energy-efficient network design is to find a subgraph $F$, such that: (1) $\forall\left(s_{i}, d_{i}\right)$ there is a path in $F$ and (2) $E_{\text {network }}$ is minimized.

We simplify the definition of $E_{\text {network }}$ to represent only idling, data transmission and reception costs:

$$
E_{\text {network }}=\sum_{u, e \in F} t_{\text {idle }}(u) \cdot c(u)+t_{\text {data }}(e) \cdot w(e),
$$

where $t_{\text {data }}(e)$ is the time spent in data communication. Since all $\left(s_{i}, d_{i}\right)$ are required to be in $F, c\left(s_{i}\right)=0$ and $c\left(d_{i}\right)=0$. Furthermore, $E_{\text {control }}(i), E_{\text {sleep }}(i)$ and $E_{s w}$ are ignored. This simplification is made only in this section to understand the complexity of the problem.

Energy-efficient network design is essentially a nodeweighted buy-at-bulk problem, which is known to be NPhard. In the node-weighted buy-at-bulk problem [8], given an undirected graph $G$, node pairs $\left(s_{i}, d_{i}\right)$ with demands $r_{i}$, and a sub-additive cost function $f(v)$ on nodes, the goal is to find a feasible routing for each pair that minimizes the total cost. In our case, the cost function $f(v)$ is uniform over all nodes.

Node-weighted network design problems are harder to approximate than their edge-weighted versions. Essentially, reducing a node-weighted problem to an edge-weighted problem requires making the graph directed and problems on directed graphs are harder to approximate. Furthermore, special cases of node-weighted single-sink/multicommodity buy-at-bulk problem are node-weighted Steiner tree/forest problems, which are hard to approximate within an $\Omega(\log n)$ factor $[18,8]$. Therefore, energy-efficient network design has also an approximation ratio $\Omega(\log n)$.

A constant approximation algorithm, MPC (Minimum Power Configuration), has been proposed for the single-sink case [24]. However, two assumptions are made: (1) link weights are bounded by node weights (i.e., $w(e) \cdot \sum r_{i} \leq$ $\alpha \cdot c(u))$ and (2) $c\left(s_{i}\right) \neq 0$ and $c\left(d_{i}\right) \neq 0$. Executing a minimum-weight Steiner tree/forest approximation algorithm in this graph, with no node weights and with edge weights equal to $c(u)$ provides an approximation ratio of $1+\alpha$ for single-source and multi-commodity case respectively.

Next, we show that the quality of solutions generated by MPC might deviate significantly in terms of $E_{\text {network }}$. To demonstrate the impact of the first assumption, we use the network in Fig. 1, where there is one sink node, $k$ sources, and nodes $i$ and $j$. Figs. 2 and 3 show two minimum-weight Steiner trees that are potential outputs of MPC, ST1 and $S T 2$. Consider the case where each source generates one packet, link activity for one packet lasts $t_{d a t a}$ and each node stays idle for a duration of $t_{\text {idle }}$. Given $P_{t x}(u, v)=\alpha \cdot z$, $P_{r x}=P_{\text {idle }}=z, E_{\text {network }}$ of $S T 1, E_{S T 1}$, is:

$$
E_{S T 1}=1 \cdot t_{\text {idle }} \cdot z+k \cdot \frac{(k+3)}{2} \cdot t_{\text {data }} \cdot(\alpha+1) \cdot z .
$$

The second term of the equation is calculated by observing that node $k$ transmits 1 packet, node $k-1$ transmits 2 packets, and node $l$ transmits $k-l+1$ packets. The relay node $i$ transmits $k$ packets. Therefore, there is a total of $k \cdot \frac{k+3}{2}$ transmissions.

$$
\begin{aligned}
& \text { Similarly, } E_{\text {network }} \text { of } S T 2, E_{S T 2} \text {, is: } \\
& \qquad E_{S T 2}=1 \cdot t_{\text {idle }} \cdot z+2 \cdot k \cdot t_{\text {data }} \cdot(\alpha+1) \cdot z .
\end{aligned}
$$

Again, the second term of the equation is calculated by observing that $k$ sources transmit one packet and the relay node $j$ transmits $k$ packets.

Comparing $E_{S T 1}$ to $E_{S T 2}$, we see that idling costs for both trees are the same $\left(1 \cdot t_{i d l e}\right)$. However, communication costs deviate with the number of sources (i.e., deviates by $\frac{k+3}{4}$ ). Essentially, while in $S T 2$ all flows are able to route through shortest paths, in $S T 1$ flows are forced to use longer routes. This shows that the structure of a Steiner tree and its impact on communication needs to be taken into account.

Next, we extend our argument to multi-commodity case and show the impact of the second assumption on performance. Fig. 4 depicts the network, where there are $k$ source and destination pairs, $\left(S_{i}, D_{i}\right), 1 \leq i \leq k$ and $S_{0}$ is the center node. Figs. 5 and 6 show two Steiner forests, $S F 1$ and $S F 2$, respectively, which are found by extending the main idea of MPC to a Steiner forest.

Next, we evaluate $S F 1$ and $S F 2$ in terms of $E_{\text {network. }}$ Given $P_{t x}(u, v)=\alpha \cdot z, P_{r x}=P_{\text {idle }}=z, E_{\text {network }}$ of $S F 1, E_{S F 1}$, is:

$$
E_{S F 1}=k \cdot t_{\text {idle }} \cdot z+2 \cdot k \cdot t_{\text {data }} \cdot(\alpha+1) \cdot z .
$$

The first term accounts for $k$ nodes that remain active to serve the flows, while the second term accounts for $2 \cdot k$ transmissions and receptions. Similarly, $E_{\text {network }}$ of $S F 2$, $E_{S F 2}$, is:

$$
E_{S F 1}=1 \cdot t_{\text {idle }} \cdot z+2 \cdot k \cdot t_{\text {data }} \cdot(\alpha+1) \cdot z .
$$




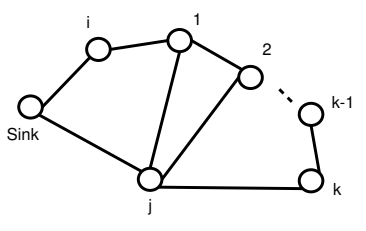

Figure 1. Network.

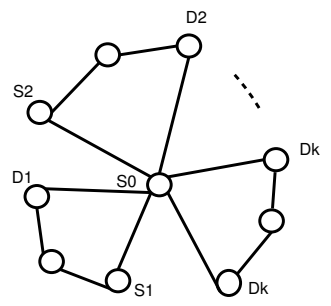

Figure 4. Network.

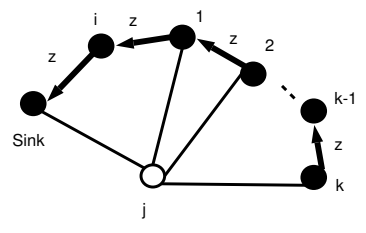

Figure 2. ST 1.

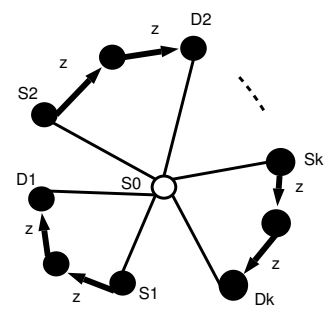

Figure 5. SF 1.

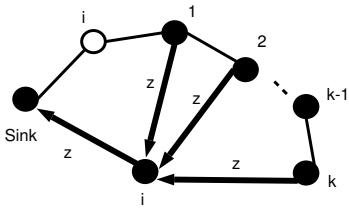

Figure 3. ST 2.

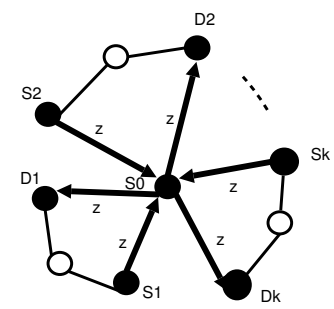

Figure 6. SF 2.
In this example both trees have the same communication costs $\left(2 \cdot k \cdot t_{\text {data }} \cdot(\alpha+1) \cdot z\right)$. However, while $S F 1$ uses $k$ relays, $S F 2$ uses only 1 relay. If the idling costs of source and destination were included in $E_{\text {network }}$, then a constant ratio of $\frac{3 \cdot k}{2 \cdot k+1}$ would be obtained.

From this discussion it is obvious that different assumptions about node and edge weights lead to different approximation bounds for the energy-efficient network design problem. However, our discussion makes some assumptions that do not hold in practice. For instance, the traffic demand, typically, cannot be determined in advance and a centralized solution is not acceptable. Therefore, next, we study three heuristics approaches that are not limited by these factors.

\section{Heuristic Approaches}

Interpreting energy-efficient network design as a multiobjective optimization problem opens the door for different heuristic approaches. Given the two objectives of minimizing communication and idling energy, we can either prioritize one objective over the other or treat them equally. Hence, we study three natural heuristics: (1) minimize communication energy first, (2) jointly optimize communication and idling energy and (3) minimize idling energy first. To implement these heuristics, we build on the fact that network design and routing are closely intertwined. Therefore, in all three approaches, information from power control and power management mechanisms is used for route selection. Consequently, route selection affects the operation of power control and power management mechanisms, hence, energy conservation. Next, we discuss these approaches in detail highlighting their benefits and drawbacks.

\subsection{Minimize Communication Energy First}

Since transmission power attenuates polynomially with distance, multiple transmissions over short distances are expected to save energy in comparison to one direct transmission. Therefore, we first consider energy-aware routing using power control as the primary optimization technique. Once the relays that minimize transmission costs are chosen, the rest of the nodes switch to a power-save mode through power management.

We implement this approach using MTPR and ODPM. Hence, the relay nodes are chosen by MTPR, and ODPM keeps these nodes in AM as long as they are actively forwarding. When the keep-alive timer of a node expires due to idling, ODPM switches the node back to PSM. We experiment with two flavors of MTPR: MTPR and MTPR+, with the following cost functions:

$$
\begin{aligned}
M T P R: f(u, v) & =P_{t}(u, v), \\
M T P R+: f(u, v) & =P_{\text {base }}+P_{t}(u, v)+P_{r x},
\end{aligned}
$$

where $P_{t}(u, v)$ is determined at transmission time using the RTS-CTS exchange at the MAC layer.

MTPR is implemented as a reactive protocol, similar to DSR [17]. The cost of the route, which is the sum of the $f(u, v)$ 's, is stored as an extra field in route requests (RREQ). When a node $v$ receives a RREQ, it updates the cost using $f(u, v)$ and rebroadcasts the packet. RREQs may 
be rebroadcast and multiple route replies (RREPs) may be sent, if they advertise a lower cost. In addition to route discovery, routing tables must be modified to store the energy cost of each route.

This approach tries to find routes with lower communication costs at the cost of increased routing overhead. However, in addition to this trade-off, idling energy is also expected to increase, which might impair the benefits of this approach.

\subsection{Joint Optimization}

In this section, power control and power management are utilized with equal emphasis. Given two routes with the same number of relays, total energy consumption is minimized when the route with minimum communication cost, which is determined by the $P_{t}(u, v)$ 's, is chosen. Hence, a natural cost function is $h(u, v)$ [24]:

$$
h\left(u, v, r_{i}\right)= \begin{cases}c(u, v), & \text { if } u \text { in AM } \\ c(u, v)+P_{\text {idle }}, & \text { if } u \text { in PSM }\end{cases}
$$

where $c(u, v)=\left(P_{t x}(u v)+P_{r x}-2 \cdot P_{i d l e}\right) \cdot \frac{r_{i}}{B}, B$ is the bandwidth and $r_{i}$ is the rate of the new flow that goes over $u-v$, if $u-v$ is on the path from $s_{i}$ to $d_{i}$. When the rate information is not available, $h\left(u, v, r_{i}\right)$ is modified by setting $\frac{r_{i}}{B}=1$. Next, we describe how $h\left(u, v, r_{i}\right)$ can be applied to proactive and reactive routing.

To use $h\left(u, v, r_{i}\right)$ in proactive routing, it is necessary to modify the routing table structure and the route look-up. Each node needs to keep information about the power management state of its neighbors and the transmit power levels to reach each neighbor. If available, $s_{i}$ includes $r_{i}$ in each packet header, and so, there is no need to maintain $r_{i}$ in the routing tables. Obviously, multiple entries for each destination might exist. When forwarding a packet, node $u$ chooses the best next-hop node $v$ with minimum $h\left(u, v, r_{i}\right)$. We implement these modifications based on the DSDV (Destination Sequence Distance Vector) routing protocol [20], similar to MPC [24]. However, the MPC implementation is limited to a single-sink scenario. Furthermore, due to MPC's routing table structure, a route update is needed whenever the flow rates change. In our implementation, a route update is only needed when the quality of a link or the power management state of a node changes. Therefore, we do not consider MPC in our evaluations.

To use $h\left(u, v, r_{i}\right)$ in reactive routing, it is necessary to modify route discovery and routing table maintenance. The modifications are similar to ones in MTPR. Different than MTPR, when a node receives a RREQ, it updates the cost of the route using the transmit power level and its power management state.

In both reactive and proactive implementations, jointoptimization tries to explore paths with less energy con- sumption at the cost of increased routing overhead. Therefore, it is susceptible to producing an overwhelming amount of control traffic to track cost changes.

\subsection{Minimize Idling Energy First}

The final approach first minimizes idling costs and second communication costs. Hence, relays are determined using power management as a primary optimization. Next, selected nodes stay active and use TPC to minimize communication costs to reach their neighbors.

We implement two variants of our approach. In the first variant, the relays are simply determined by a reactive shortest-path routing algorithm, which is DSR in our implementation. Once a node is chosen as a relay, ODPM maintains the power management state of the node based on its current participation in routing. In the second variant, TITAN [21], is used to select relays. TITAN maintains a backbone of nodes, which addresses the shortcoming of activating redundant nodes in ODPM. The basic idea is to favor nodes that are already in AM as good candidates for routing, so that the nodes in PSM can continue sleeping. Therefore, in TITAN, a node probabilistically participates in route discovery based on the its power-management state, size of its neighborhood and the number of backbone nodes it knows of. Hence, as route diversity in the network increases, TITAN reduces the number of relays.

Our approach provides a low-complexity solution to energy-efficient network design problem. Essentially, this low complexity is the key to achieving high energy savings without degrading communication performance.

\section{Performance Evaluation}

In this section, we present the first comprehensive study of the energy-efficient network design problem by investigating the three heuristic approaches introduced in Section 4. Through an analytical study, we first rule out the first approach (i.e., minimize communication energy first) showing that it does not provide energy savings with wellresearched wireless cards. Next, we evaluate the second and third approaches (i.e., joint optimization and power management as primary optimization) via a simulation study. Finally, we extend our simulation study to a hypothetical wireless card to evaluate if the first approach has any potential for energy savings in comparison to the second and third approaches.

\subsection{Analytical Study}

In this section, our goal is to understand the effectiveness of power control as a primary optimization. To this end, we analyze the energy savings through using relays 
between two nodes that are in transmission range of each other, essentially, revealing when using relays saves energy in comparison to direct transmission.

The steps to derive the optimal number of relays (i.e., optimal hop count) are similar to calculating the characteristic distance $[6,12]$, which is the optimal hop distance that minimizes the energy cost of end-to-end transmission. However, in [6] the effect of $E_{\text {passive }}(i)$ is ignored and in [12] the relationship between transmission range and characteristic distance is not considered. Essentially, characteristic distance might be greater than the transmission range, in which case only direct transmission is feasible. To capture this effect, we define the characteristic hop count as the optimal number of hops between two nodes that can also trannsmit directly. Obviously, the characteristic hop count must be greater than two to save energy through relays.

To derive the characteristic hop count, we analyze the total energy consumption on a route, $E_{r}$. Using equations from Section 2.1,

$$
E_{r}=\sum_{i}^{m+1} E_{\text {comm }}(i)+E_{\text {passive }}(i),
$$

where $i=1$ is the source and $i=m+1$ is the destination, and there are $m-1$ relays. We assume that the nodes on the route are in AM, and hence, $t_{\text {sleep }}=0$ and $E_{s w}=0$. For a given rate $R$, bandwidth $B$, and time $t, t_{r x}^{\text {data }}(i)=$ $t_{t x}^{d a t a}(i)=\frac{R}{B} \cdot t$, except $t_{r x}^{\text {data }}(1)=0$ and $t_{t x}^{\text {data }}(m+1)=0$. The remainder of $t$ is spent in idling. Ignoring $E_{\text {control }}(i)$,

$E_{r}=\frac{R}{B} \cdot t \cdot \sum_{i=1}^{m} P_{t x}\left(d_{i}\right)+m \cdot P_{r x}+\left(m+1-2 \cdot m \cdot \frac{R}{B}\right) \cdot t \cdot P_{i d l e}$.

$P_{t x}\left(d_{i}\right)$ denotes the transmission cost between two nodes separated by $d_{i}$. Assuming a $1 / d^{n}$ path loss, $P_{t x}(d)$ can be modeled as $P_{t x}(d)=P_{\text {base }}+\alpha_{2} \cdot d^{n}$, where $\alpha_{2} \cdot d^{n}$ represents $P_{t}(i, j)$, and $\alpha_{2}$ is the power to drive the transmitter amplifier [6]. Since $E_{r}$ is convex, it is minimized when all hop distances are equal. Therefore, using $d_{i}=D / m$, we find optimal hop count, $m_{\text {opt }}$, by solving $\frac{\partial E_{\text {route }}}{\partial m}=0$ :

$$
m_{\text {opt }}=\sqrt[n]{\frac{(n-1) \cdot \alpha_{2}}{P_{\text {base }}+P_{r x}+\frac{1-2(R / B)}{R / B} \cdot P_{\text {idle }}}} \cdot D .
$$

Since the characteristic hop count is an integral value, it is $\left\lceil m_{\text {opt }}\right\rceil$ if $m_{\text {opt }}<1$, and $\left\lfloor m_{\text {opt }}\right\rfloor$ if $m_{\text {opt }} \geq 1$.

By definition, only $\left\lfloor m_{\text {opt }}\right\rfloor \geq 2$ justifies using relays between two nodes that are in transmission range. We plot $m_{\text {opt }}$ for four different wireless cards as $R / B$ increases (see Fig. 7). The radio model for each wireless card is given in Table 1. We use the existing models for Cabletron [19] and LEACH cards [15]. The models for Aironet 350 and Mica2 [10] are derived using polynomial curve fitting on existing measurement studies. In Fig. 7, since $m_{\text {opt }}<2$
Table 1. Radio parameters for four wireless

\begin{tabular}{|c|c|c|c|}
\hline Cards & $P_{\text {idle }}$ & $P_{r x}$ & $\overline{P_{t x}(d(m))}$ \\
\hline 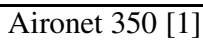 & 1350 & 1350 & $2165+3.6 \cdot 10^{-7} \cdot d^{4}$ \\
\hline Cabletron [9] & 830 & 1000 & $1118+7.2 \cdot 10^{-8} \cdot d^{4}$ \\
\hline Hypothetical & & & $1118+5.2 \cdot 10^{-6} \cdot d^{4}$ \\
\hline Mica2 [10] & 21 & 21 & $10.2+9.4 \cdot 10^{-7} \cdot d^{4}$ \\
\hline LEACH [15] & $x \cdot 50$ & 50 & $\begin{array}{c}50+1.3 \cdot 10^{-6} \cdot d^{4} \\
50+10^{-2} \cdot d^{2}\end{array}$ \\
\hline
\end{tabular}
cards $(m W)$

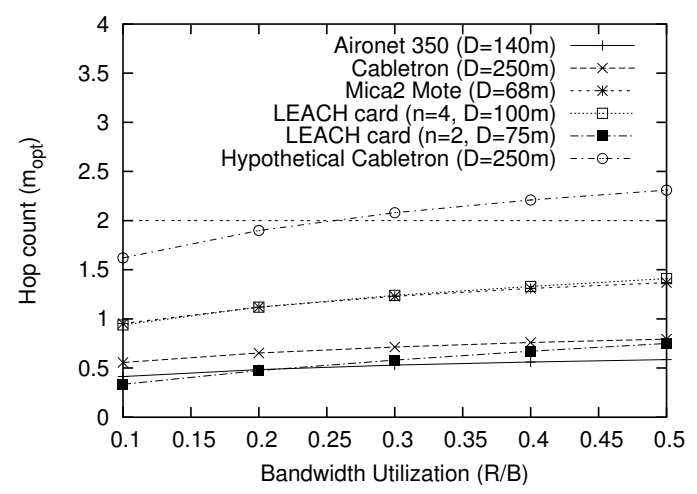

Figure 7. $m_{\text {opt }}$ for different cards.

for all rates, only direct transmission is feasible. The case when the bandwidth is fully utilized (i.e., $R / B=0.5$ ) corresponds to the case when $P_{\text {idle }}$ has no effect. Therefore, even when idling energy is not accounted for, direct transmission is more energy-efficient than communicating through relays.

Power control as an optimization metric is meaningful only for cards with certain characteristics. For instance, setting $\alpha_{2} \geq 5.16 \cdot 10^{-6}$ for Cabletron satisfies $m_{\text {opt }} \geq 2$ for $R / B=0.25$ (see Hypothetical Cabletron in Fig. 7). Consequently, the transmit power to reach $D=250 \mathrm{~m}$ increases up to $20 \mathrm{~W}$, which cannot be supported due to $1 \mathrm{~W}$ limit by the FCC in the USA [4], and $100 \mathrm{~mW}$ limit by ETSI in Europe [2]. Given these limitations, we are not aware of any card that satisfies $m_{o p t} \geq 2$. Furthermore, new cards consume less energy in transmit than receive [16], which makes using relays between two nodes in transmission range even more questionable. Nevertheless, in Section 5.2.3, we evaluate all approaches with the Hypothetical Cabletron to provide a thorough study of the trade-offs.

In this section, we have shown that energy-aware routing that uses additional relays between two nodes (like PARO [14]) does not save energy. However, we discuss power control only from an energy perspective. Power control affects also spatial reuse, especially when the commu- 
nication consists mostly of one-hop flows. However, its advantage in the presence of multi-hop flows is not obvious. We will study the trade-off between energy and spatial reuse as future work.

\subsection{Simulation Study}

The goal of our simulation study is to understand the effectiveness of the heuristic approaches in various network settings. We use delivery ratio to measure communication performance, which is the ratio of the number of received data packets to the number of sent data packets. The performance in terms of energy is evaluated by energy goodput, which is the ratio of total application bits delivered to the total energy consumed (i.e., $E_{\text {network }}$ ).

We use ns2 [3] for our simulations. In Sections 5.2.1 and 5.2.2, we present results with the Cabletron card (see Table 1). We simulate the following protocols: proactive joint optimization (DSDVH-ODPM), reactive joint optimization with and without traffic rate information (DSRHODPM(rate) and DSRH-ODPM(norate)), and power management as primary optimization (DSR-ODPM-PC and TITAN-PC). We use two baselines: DSR with power management (DSR-ODPM) and DSR with all nodes active (DSR-Active). Section 5.2.3 presents results with the Hypothetical Cabletron card, described in Section 4.1. In this section, we evaluate the performance of power control as primary optimization (MTPR and MTPR+).

ODPM uses IEEE-802.11 PSM for sleep scheduling. For PSM, the beacon interval is $0.3 \mathrm{~s}$ and the ATIM window is $0.02 \mathrm{~s}$, as suggested in [9]. Both the beacon and the ATIM intervals are long enough to compensate for the cost of switching between sleep and idle states. For ODPM, the keep-alive timers are set to $10 \mathrm{~s}$ for RREPs and $5 \mathrm{~s}$ for data messages. For both cards, transmit power levels are assumed to be infinitely adjustable. Although this is optimistic, it permits us to concentrate on the trade-offs in energy-efficient network design.

\subsubsection{Small Networks}

In these simulations, 50 nodes are placed, uniformly at random, in a $500 \times 500 \mathrm{~m}^{2}$ static network. There are $10 \mathrm{CBR}$ flows. The packet size is $128 \mathrm{~B}$. The start time of each flow is random between $20 \mathrm{~s}$ and $25 \mathrm{~s}$. Each simulation runs for 900 s. Each graph depicts an average of 5 runs and $95 \%$ confidence intervals.

To understand the impact of traffic load, we evaluate performance as the traffic rate of each flow increases between 2-6 Kbit/s (i.e., 2-6 packets/s). Except DSDVH-ODPM, all approaches use approximately the same number of relays ( $\approx 26$ nodes), and so, their energy goodput performance is similar (see Fig. 9). Furthermore, even though

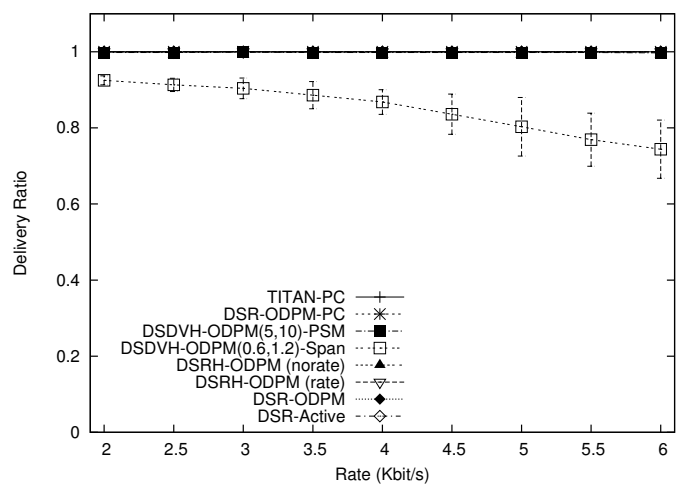

Figure 8. Delivery ration: $500 \times 500 \mathrm{~m}^{2}$.

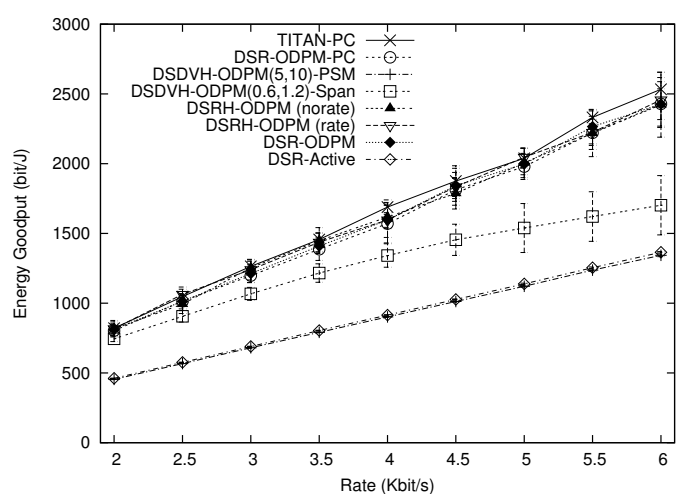

Figure 9. Energy goodput: $500 \times 500 \mathrm{~m}^{2}$.

the transmit energy significantly reduces with power control (ODPM uses 54-59\% more transmit energy than TITAN), its effect on the total is not visible since $\sum_{i} E_{\text {passive }}(i) \gg$ $\sum_{i} E_{\text {comm }}(i)$ (see Fig. 9 and 500x500 lines in Fig. 10).

Compared to the rest, DSDVH-ODPM has significantly worse energy goodput (e.g., $\approx 85 \%$ lower than TITANPC) due to routing table updates. Furthermore, in IEEE 802.11 PSM, these updates keep nodes awake for an entire beacon interval, increasing idling energy consumption. Hence, DSR-Active and DSDVH-ODPM have almost the same energy goodput in Fig. 9 (their lines overlap). Therefore, we evaluate the following improvements for PSM [9]: (1) advertising each broadcast message and (2) using an advertised traffic window so that a node can sleep after receiving all advertised messages. We also reduce the keepalive timer for data packets to $0.6 \mathrm{~s}$ (i.e., two beacon intervals) and RREPs to $1.2 \mathrm{~s}$. This version of DSDVHODPM is labeled DSDVH-ODPM(0.6,1.2)-Span. As expected, the energy goodput in this case is higher (e.g., now $10 \%-49 \%$ worse than TITAN-PC). However, the advertised traffic window has a side-effect of reducing de- 


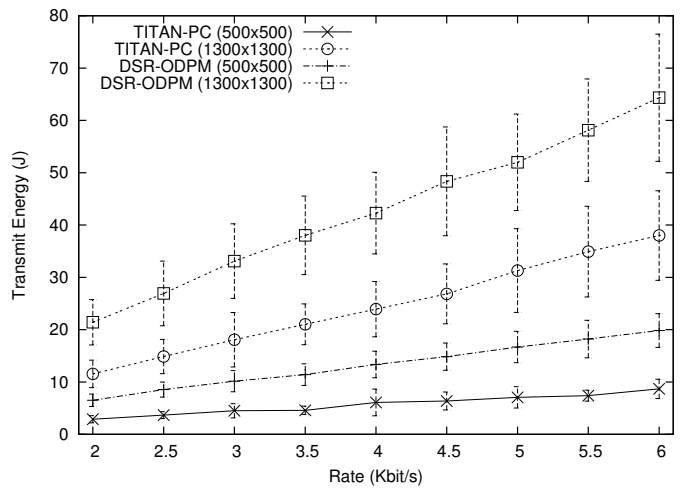

Figure 10. Transmit energy.

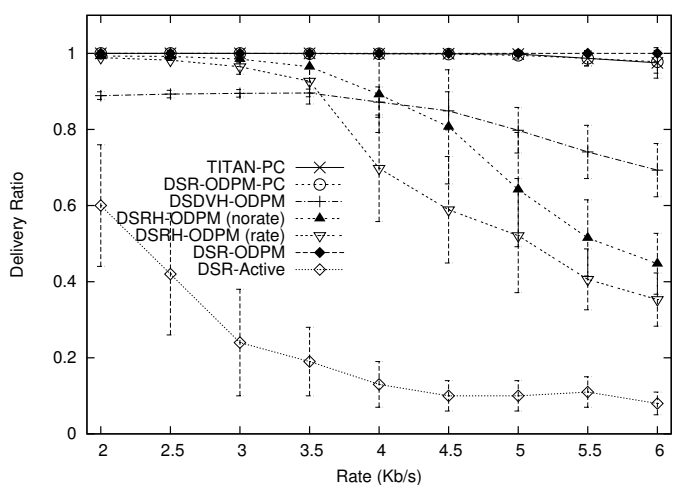

Figure 11. Delivery ratio: $1300 \times 1300 \mathrm{~m}^{2}$.

livery ratio: DSDVH-ODPM(0.6,1.2)-Span delivers 74\%$92 \%$, while the rest delivers $100 \%$ (see Fig. 9). These results show that depending on the routing protocol the overhead of joint optimization becomes visible even in small networks.

\subsubsection{Large Networks}

To evaluate the scalability of the protocols, we next present simulation results in larger networks. We simulate 200 nodes placed uniformly at random, in a $1300 \times 1300 \mathrm{~m}^{2}$ static network. There are 20 CBR flows. The start time for each flow is random between $20 \mathrm{~s}$ and $25 \mathrm{~s}$. Each simulation runs for $600 \mathrm{~s}$. Each graph depicts an average of 10 runs and 95\% confidence intervals.

In contrast to small networks, the differences among approaches are now evident (see Figs. 11 and 12). Power management as primary optimization performs significantly better than joint optimization. There is a threshold rate, $3.5 \mathrm{~Kb} / \mathrm{s}$, where joint optimization starts performing with high deviation. Essentially, as the rate increases, the control

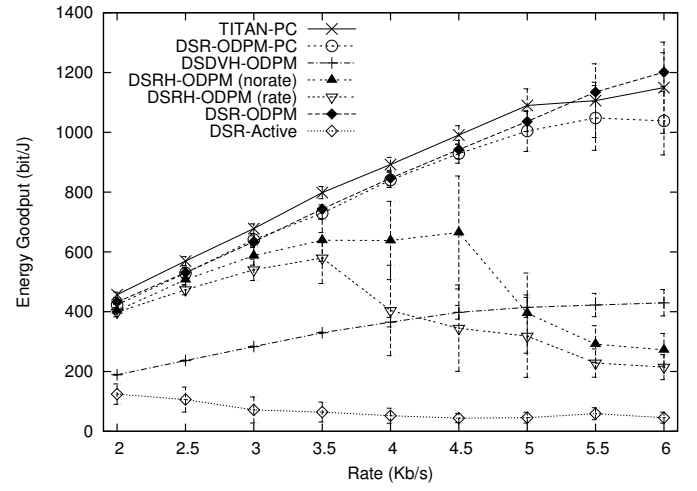

Figure 12. Energy goodput: $1300 \times 1300 \mathrm{~m}^{2}$.

Table 2. Performance with node density

\begin{tabular}{|l|c|c|}
\hline \# of nodes & DSR-ODPM-PC & TITAN-PC \\
\hline \multicolumn{3}{|c|}{ Delivery Ratio } \\
\hline 300 & $0.933 \pm 0.056$ & $0.993 \pm 0.004$ \\
\hline 400 & $0.405 \pm 0.093$ & $0.923 \pm 0.102$ \\
\hline \multicolumn{3}{|c|}{ Energy Goodput (bit/J) } \\
\hline 300 & $507.162 \pm 137.267$ & $674.381 \pm 37.084$ \\
\hline 400 & $90.711 \pm 22.278$ & $929.812 \pm 278.794$ \\
\hline
\end{tabular}

overhead of both proactive and reactive protocols interferes with data communication.

It is also observed that power management has a positive effect on scalability. In all heuristic approaches, power management imposes a scheduling on transmissions and receptions through beacon and ATIM intervals. In the absence of such a scheduling, DSR-Active's scalability is significantly impaired (see Figs. 11 and 12). On the other hand, the effect of power control is not obvious, even though TITAN-PC indeed incurs less transmit energy (66-86\% less than ODPM, see Fig. 10).

The evaluations up to this point show that DSR-ODPMPC and TITAN-PC perform similarly. Therefore, we further evaluate these two protocols with different node densities, setting the per-flow traffic rate to $4 \mathrm{~Kb} / \mathrm{s}$ and without changing the positions of source and destination nodes. The results show that TITAN-PC can scale to denser networks (see Table 2). Essentially, the routing overhead of DSR-ODPMPC explodes with network density, and TITAN is less affected since active nodes dominate route discovery. Hence, as the node density increases, TITAN-PC is the only protocol that can maintain high performance.

\subsubsection{Hypothetical Networks}

In this section, we evaluate all protocols with the Hypothetical Cabletron card. The goal of our study is to understand the trade-offs among all three heuristics when power con- 


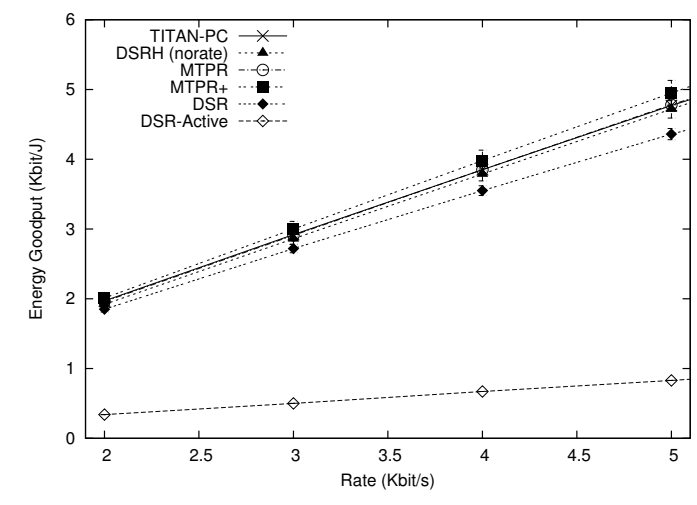

Figure 13. Energy goodput for low traffic rates with perfect sleep scheduling.

trol as primary optimization has potential to save energy. For the sake of clarity of graphs, we omit the results for DSR-PC and DSRH(rate).

To allow all protocols to exhibit their characteristic behavior (e.g., MTPR favors short hop distances and TITAN focuses traffic on backbone nodes), we simulate a static $300 \times 300 \mathrm{~m}^{2}$ network with 49 nodes placed on a $7 \times 7$ grid. There are 7 CBR flows, where a source on the left side sends to a destination on the right side. The start time for each flow is random between 20 s and 25 s. Each simulation is $900 \mathrm{~s}$. We simulate per-flow traffic rates between $2 \mathrm{Kbit} / \mathrm{s}-200 \mathrm{Kbit} / \mathrm{s}$ (above $200 \mathrm{Kbit} / \mathrm{s}$ is beyond node capacity). To understand the potential of each approach without the side effects of high rates (e.g., packet losses due to buffer overflows), we find the time when the routes stabilize for the $2 \mathrm{Kbit} / \mathrm{s}$ and use these routes to calculate $E_{\text {network }}$ for higher rates.

We evaluate two sleep scheduling strategies: (1) perfect sleep scheduling, where nodes wake up at the exact time when they are needed, and (2) ODPM scheduling, where active nodes are always idling in the expectation of traffic, while the rest of the nodes are asleep.

Figs. 13 and 14 show energy goodput for low traffic rates $(2 \mathrm{~K}-5 \mathrm{~K})$. As expected, with perfect sleep scheduling, all protocols perform similar with the exception of DSRActive. However, the energy goodput of all protocols degrades with ODPM. When the traffic load is low, we see energy savings mostly from reducing the number of relays. Therefore, with ODPM scheduling, TITAN outperforms other protocols.

Figs. 15 and 16 show energy goodput for high traffic rates. With no idling costs, TITAN-PC achieves lower energy goodput compared to MTPR, MTPR+ and DSRH (norate). Essentially, using longer links becomes more expensive as the rate increases. However, with ODPM schedul-

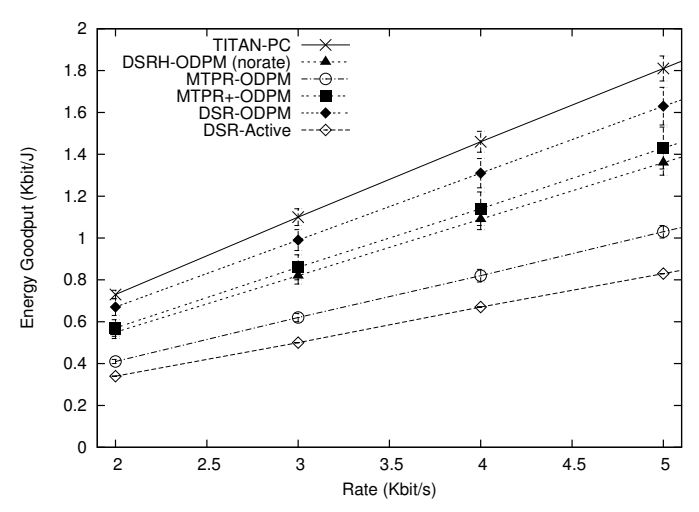

Figure 14. Energy goodput for low traffic rates with ODPM scheduling.

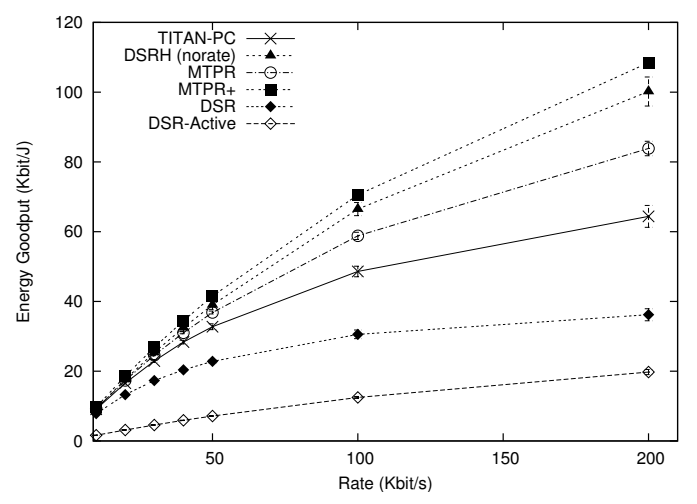

Figure 15. Energy goodput for high traffic rates with perfect sleep scheduling.

ing, TITAN-PC outperforms others protocols for rates below $200 \mathrm{Kbit} / \mathrm{s}$. Furthermore, the difference in performance at $200 \mathrm{Kbit} / \mathrm{s}$ is less pronounced compared to Fig. 15.

These results show that even with a hypothetical card and ideal scenarios, power control as primary optimization and joint optimization provide high energy savings only with perfect sleep scheduling. When idling costs are taken into account, these approaches outperform power management as primary optimization at only very high bandwidth utilization. However, it is not clear if such high rates can be supported in multi-hop wireless networks due to potentially high contention and delay. Therefore, our approach will remain valuable unless the characteristics of wireless cards change and perfect sleep scheduling becomes feasible. 


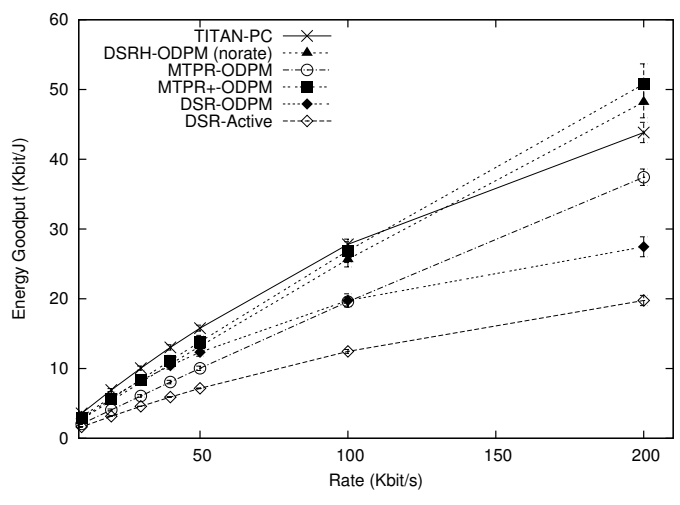

Figure 16. Energy goodput for high traffic rates with ODPM scheduling.

\section{Conclusion}

Saving energy from wireless communication requires reducing energy consumption for all radio states. In this paper, we present this problem as an energy-efficient network design problem and study three heuristic approaches. Evaluation results show that our approach that gives priority to idling energy consumption achieves the desired property of being both bandwidth and energy-efficient. Furthermore, our study exposes a commonly held myth about the potential energy savings from power control as a primary energy conservation technique.

In this work, we mainly consider minimizing instantaneous network energy consumption, which does not necessarily translate into longer network lifetime. The lifetime of a wireless network is dependent on many factors such as the type of application, network traffic, number of nodes, available energy, path loss and radio energy parameters [6]. Due to the complexity of the energy-efficient network design problem, incorporating such lifetime constraints defined by the application is part of our future work.

\section{References}

[1] Cisco Aironet 350. http: //www. cisco.com/en/US / products/hw/wireless/ps 4555.

[2] ETSI EN 300 328. http: / / www.etsi.gov/.

[3] Ns2. http://www.isi.edu/nsnam/ns.

[4] Title 47 of the code of federal regulations (CFR) part 15. http://www.fcc.gov/oet/info/rules.

[5] Wireless LAN medium access control (MAC) and physical layer (PHY) specifications. IEEE Std. 802.11, 1999.

[6] M. Bhardwaj, T. Garnett, and A. P. Chandrakasan. Upper bounds on the lifetime of sensor networks. In ICC, 2001.

[7] J.-H. Chang and L. Tassiulas. Maximum lifetime routing in wireless sensor networks. IEEE/ACM Transactions on Networking, 12(4):609-619, 2004.
[8] C. Chekuri, M. T. Hajiaghayi, G. Kortsarz, and M. R. Salavatipour. Approximation algorithms for node-weighted buy-at-bulk network design. In ACM-SIAM SODA, 2007.

[9] B. Chen, K. Jamieson, H. Balakrishnan, and R. Morris. Span: An energy-efficient coordination algorithm for topology maintenance in ad hoc wireless networks. In MobiCom, 2001.

[10] A. Falchi. Sensor networks: performance measurements with motes technology. Technical Report etd-05252004154652, University of Pisa, 2004.

[11] L. M. Feeney and M. Nilsson. Investigating the energy consumption of a wireless network interface in an ad hoc networking environment. In INFOCOM, 2001.

[12] Q. Gao, K. J. Blow, D. J. Holding, I. W. Marshall, and X. H. Peng. Radio range adjustment for energy-efficient wireless sensor networks. Ad Hoc Networks, 4(1):75-82, 2006.

[13] M. X. Goemans and D. P. Williamson. Approximation Algorithms for NP-hard problems, chapter The Primal-Dual Method for Approximation Algorithms and Its Application to Network Design Problems. 1997.

[14] J. Gomez, A. T. Campbell, M. Naghshineh, and C. Bisdikian. PARO: supporting dynamic power controlled routing in wireless ad hoc networks. Wireless Networks, 9(5):443460, 2003.

[15] W. B. Heinzelman. Application-Specific Protocol Architectures for Wireless Networks. PhD thesis, MIT, 2000.

[16] I. Howitt, R. Neto, J. Wang, and J. M. Conrad. Extended energy model for the low rate WPAN. In MASS, 2005.

[17] D. B. Johnson, D. A. Maltz, and J. Brosch. Ad Hoc Networking, chapter DSR: The Dynamic Source Routing Protocol for Multi-Hop Wireless Ad Hoc Networks, pages 139-172. 2001.

[18] P. Klein and R. Ravi. A nearly best-possible approximation algorithm for node-weighted steiner trees. Journal of Algorithms, 19(1):104-115, 1995.

[19] S.-J. Park and R. Sivakumar. Load-sensitive power control in wireless ad hoc networks. In GLOBECOM, 2002.

[20] C. Perkins and P. Bhagwat. Highly dynamic destinationsequenced distance vector routing (DSDV) for mobile computers. In SIGCOMM, 1994.

[21] C. Sengul and R. Kravets. Conserving energy with ondemand topology management. In MASS, 2005.

[22] C. Sengul and R. Kravets. On integrating energy conservation approaches for ad hoc networks. Refereed poster in MobiHoc, May 2005.

[23] S. Singh, M. Woo, and C. S. Raghavendra. Power-aware routing in mobile ad hoc networks. In MobiCom, 1998.

[24] G. Xing, C. Lu, Y. Zhang, Q. Huang, and R. Pless. Minimum power configuration in wireless sensor networks. In MobiHoc, 2005.

[25] R. Zheng and R. Kravets. On-demand power management for ad hoc networks. In INFOCOM, 2003. 